\title{
Linguistic analysis of suicide notes in Spain
}

\author{
Mercedes Fernández-Cabanaa ${ }^{\mathrm{a}, \star}$ \\ Julio Jiménez-Féliz ${ }^{\mathrm{b}}$ \\ María Teresa Alves-Pérez ${ }^{c}$ \\ Raimundo Mateos ${ }^{\mathrm{a}}$ \\ Ignacio Gómez-Reino Rodríguez ${ }^{\mathrm{d}}$ \\ Alejandro García-Caballero ${ }^{\mathrm{a}, \mathrm{d}}$ \\ a Department of Psychiatry, School of \\ Medicine, University of Santiago de \\ Compostela, Praza do Obradoiro, \\ Santiago de Compostela 15782 \\ b Institute of Legal Medicine of Galicia \\ (IMELGA), Ourense \\ c Universidade de Vigo, NeCom \\ Research Group \\ d Complexo Hospitalario Universitario \\ de Ourense, Ourense
}

SPAIN

\begin{abstract}
Background and Objectives: Text analysis software like "Linguistic Inquiry and Word Count" (LIWC) has been used for the analysis of suicide notes and suicidal texts in English. This is the first analysis of suicide notes using this method in Spanish and, as far as we know, its first application to suicide notes in Europe.

To compare the sociodemographic and forensic characteristics of a consecutive sample of suicide victims studying the differences between those who left suicidal note and those who did not. To study a sample of suicidal notes from Spain using LIWC, comparing it's linguistic features by gender, age and environment.

Methods: 144 consecutive suicide cases were analyzed. 23 suicide notes obtained from this sample were processed using LIWC, the results were compared by gender, age and environment of the author.

Results: People who left suicide notes were younger than non- writers; more frequently single, divorced or widowed and emotional troubles were reported as frequent triggers. Suicide notes written by women were significantly longer, had more emotional content, tentative expressions, denials, pronouns in first person plural and verbs in past and future tenses. Urban cases showed higher emotional expression and word complexity whereas rural cases showed a higher use of social words.

Conclusions: Our study shows some differences between people who left suicide note and those who didn't and confirms the LIWC ability to detect differences in suicidal speech by gender and by the rural/ urban background of its authors.
\end{abstract}




\section{Introduction}

Research on suicidal behavior has paid special attention to suicide notes. Beside their obvious forensic interest, from the psychological point of view they are a valuable tool for deciphering the suicidal mind ${ }^{1,2}$.

The occurrence of suicide notes varies, ranging from $15 \%$ to $38.1 \%$ of those who die by suicide ${ }^{3}$. Analysis of suicide notes has followed two main streams. On one side, researchers study the psychosocial and clinical profile of authors, or compare them with suicide victims who didn't leave notes. On the other side the researchers analyze qualitative features of writing (content and topics covered, comparison of real notes vs. fake ones).

An analysis of 40 suicide notes from India found that a majority of note writers were young males, (ranging from 21 to 30 years), and committed suicide at home by hanging; being depression and hopelessness the most common diagnosis ${ }^{4}$. Among 621 suicide victims from USA, $231(37.2 \%)$ left a suicide note. In this sample, note writers were more frequently living alone and were less likely to have made previous suicide threats ${ }^{3}$. Another study ${ }^{5}$ analyzing 1,051 cases of suicide from Australia, found a $33.1 \%$ of suicide notes. Note writers were younger, divorced or living alone, and less supervised by primary care or mental health services. They were suffering severe stress, and showed more frequent hypochondriac traits and less frequent psychotic symptoms. Regarding the causes, those motivated by extrapunitive reasons and interpersonal loss or conflict were more likely to write a suicide note, while those motivated by a psychiatric disturbance were less prone to write. Finally suicidal methods like gas poisoning, firearms or medication were more frequent among note writers.
As we can see, these disparate data question the suitability of drawing conclusions from suicide notes and suicide note writers to non-writers. This is an unresolved issue to date and reminds us the necessity to bear in mind cultural differences to approach these data. To our knowledge, there have been no previous studies in our community to compare the characteristics of people who leave suicide note with those who did not.

Regarding topic analysis, qualitative techniques have been used, following two major paths. Foster ${ }^{6}$ for instance searched for different themes in the texts, finding "apology / shame" (in $74 \%$ of suicide notes), "love for those left" (in $60 \%$ of them), "life too much to bear" (48\%), "instructions on practical issues post mortem" (36\%), "hopelessness/nothing to live for" $(21 \%)$, and "advice for those left behind" (21\%). Whereas Leenaars ${ }^{7}$ extracted the topics from different theoretical models distinguishing between intrapsychic variables (unbearable psychological pain, cognitive state of mental constriction, indirect expressions of unconscious processes, inability to adjust, and weakness of ego) and interpersonal variables (problems with interpersonal relations, rejection-aggression hypothesis, and identificationegression hypothesis) and used them to analyze suicide notes ${ }^{8}$. Another line of work was to compare real notes with fake ones. Lester ${ }^{9}$ studying the appearance of the three main reasons for suicide described by Menninger ${ }^{10}$ in 1938 (anger, guilt and desire to escape) found that anger appears more in genuine notes, while a year later a similar analysis ${ }^{11}$ reported guilt as more frequent in genuine ones.

The difficulty of finding a universal, unbiased method for analyzing content has led to automatic linguistic analysis techniques that allow the statistical processing of texts with minimal theoretical intervention. One of the most utilized tools for computerized analysis of texts is Linguistic Inquiry and Word Count, 
a software application developed by Pennebaker, Francis and Booth ${ }^{12}$. The Linguistic Inquiry and Word Count (known as LIWC) analyzes texts on 72 linguistic variables including standard language categories (articles, prepositions, pronouns, including first person singular, first person plural etc.), psychological processes (positive emotions, negative cognitive processes, etc.), words related to relativity (time, tense, space, etc.), and content categories (sex, money, home, etc.). Many of the LIWC2001 categories are arranged hierarchically, and each word or word stem found in the text belongs to one or more word categories. LIWC2001 calculates the percentage of words in the text that match each of the dimensions of language, using an inner dictionary that defines which words should be counted in the different files, except in the case of categories such as the total number of words in the text, or the number of words per sentence, of which computes this number instead of offering the result as a percentage. LIWC2001 has been validated in Spanish ${ }^{13}$ in 2007, which has resulted in an increase in the number of words included (over 7,000), due to the characteristics of the Spanish language.

The use of LIWC in suicidology has a long history. In 2001 Stirman and Pennebaker ${ }^{14}$ found linguistic differences in the work of nine suicidal poets compared to nine non-suicidal poets. LIWC has also been used to analyze the texts of other poets ${ }^{15-17}$, historical figures ${ }^{18}$ or celebrities ${ }^{19}$ died by suicide. Regarding suicide notes, notes from those who attempted suicide are linguistically different from those who completed suicide, with fewer positive emotions, fewer social references and future tense verbs, and more metaphysical references in notes from the attempted suicides ${ }^{20}$. Real and simulated suicide notes are linguistically different, with genuine notes being longer, with more pronouns, fewer prepositions and words related to causation, and more references to people and social processes ${ }^{21}$. Women use more words showing positive feelings, present tense, cognition or insight, and first person pronouns; and older writers use fewer pronouns in second person, fewer references to self or to the present, and fewer words related to cognitive processes ${ }^{22}$. All these studies, however, were on English speakers. As far as we know, no attempt has been made to analyze suicide notes with LIWC in languages other than English.

In our study we have the following aims: to determine the clinical and sociodemographic characteristics of a sample of suicide victims in our community finding possible differences between those who left suicide notes and those who didn't; and to analyze with the Spanish version of the LIWC the suicide notes collected to find out if there are linguistic differences according to gender, environment, or age of its authors.

\section{Method}

\section{Selection process}

Our sample size was determined by the number of deaths by suicide $(\mathrm{N}=144)$ occurred between July 2006 and December 2009 in the province of Ourense, located in northwest Spain (325,000 inhabitants). In all cases the death was certified as due to suicide by the Medical Examiner and studied by the Institute of Legal Medicine of Galicia (IMELGA) in Ourense. In 26 cases (18.06\%) the victim left a suicide note. Notes were collected or photographed at the scene by the Forensic Scientists or the Scientific Police and were part of the court file.

We analyzed 23 suicide notes with LIWC, given that two files were excluded from linguistic analysis, the first one because it in- 
cluded only two phone numbers (the notes that consist of mere listings or just phone numbers, or account numbers, without other displays must be usual in this type of studies, although we have not found reference to this fact in the literature), and the second one because it was written in Korean. In a third case the note could not be retrieved from the file.

\section{Procedure}

The sample of people dead by suicide was characterized in terms of its sociodemographic and forensic characteristics that included inter alia: date of suicide, age, sex, residence, marital status, stressful life events, medical and psychiatric history, drug treatment, number of previous attempts, methods of death and triggers. These data were obtained by the medical examiner following a semi-structured questionnaire with relatives and neighbors included in the research that takes part during the removal of the dead bodies. A medical examiner (second author of this paper) had direct access to the court files and carried out data collection following a predetermined protocol designed to increase its reliability. All personal data were processed according to the guidelines of the World Health Organization and the declaration of Helsinki.

We divided the total sample into two groups: those who had left suicide note, and those without suicide note, to analyze if there were sociodemographic or forensic differences between them.

Regarding linguistic analysis, we analyzed the written material collected in each case with the Spanish version of LIWC $^{13}$ as a continuous text (although in some cases there were several texts with different intention or recipients), in order to increase the number of words in each case and avoid arbitrariness in the classification. We included and processed proper names and numbers when they were embedded within the suicide notes. In two cases we had to translate the suicide notes from Galician into Spanish. Translated texts had a high number of common words between the two languages (due to reduced linguistic distance) requiring only grammatical and not lexical adaptation. The linguistic features of suicide notes were related to gender, age and background of their authors. We compared our results with those obtained in previous studies.

In the statistical analysis we used Chi square test, the test for comparison of means T-Student and the nonparametric test $\mathrm{U}$ Mann-Whitney, according to the characteristics of the variables compared.

\section{Results}

\section{Characteristics of the total sample and comparison with/without suicide note}

In the complete sample $(\mathrm{N}=144)$, the mean age was 61.77 years $(\mathrm{SD}=19.53), 104$ $(72.22 \%)$ were men, 55 (44.36\%) were married or lived in couples, and $85(64.88 \%)$ lived in rural areas $(<10,000$ inhabitants). The suicide method was hanging in 81 victims $(57.04 \%)$, poisoning in $16(11.27 \%)$, gunshot in $15(10.56 \%)$, or jumping in 15 (10.56\%). In most cases the suicide occurred at home $(41.86 \% ; \mathrm{n}=54)$, in annexes $(30.23 \% ; n=39)$ or at work places $(20.93 \%$; $\mathrm{n}=27)$. About $20 \%$ of individuals studied had a previous suicide attempt. Most frequently reported triggers were the presence of a previous mental illness $(46.24 \% ; n=43)$, deterioration of general health $(16.13 \% ; \mathrm{n}=$ $15)$, familiar $(15.05 \% ; \mathrm{n}=14)$ or emotional 
troubles $(11.83 \% ; \mathrm{n}=11)$, and, in $8.60 \%$ of the victims $(n=8)$, the presence of severe somatic disease (percentages provided in this paragraph refer to the number of valid cases for each variable depending on missing data).

In the comparison between people who left suicide note $(18.06 \%)$ and those with no note, we found significant differences ( $p=$ 0.032 ) in age, with younger individuals leaving more suicide notes (mean age of 54.38 years, $S D=20.94$ ) than those who did not (mean age of 63.42, SD =18.91). We also found a marginally significant difference $(p$ $=0.065)$ in marital status, with a higher percentage of single or similar (separated/divorced or widowed) amongst note writers; and in the choice of workplace as the place of suicide $(p=0.061)$, higher in people without suicide note. The difference in triggers reported by relatives in both groups was significant. The most common trigger in suicide note writers was the occurrence of emotional troubles $(p<0.001)$, which were reported in the $47.37 \%$ of note writers (nine cases out of nineteen) vs. just in $2.70 \%$ of non-writers (2 of the 74 remaining cases in which a trigger was reported). In the group who did not leave a suicide note the most commonly reported trigger $(54.05 \%, 40$ of the 74 individuals) was the existence of a mental illness ( $p=$ 0.006 ), only reported in $15.79 \%$ of note writers. The trigger of economic and/or work trouble was only reported in two cases of people who left suicide note $(p=0.053)$. We did not find significant differences between note writers and non-writers in terms of gender, residence or method of suicide. Compared variables are listed in Table 1. Given that not in all cases did the files include all the variables studied, we entered the number of valid cases for each feature in the table.

\section{Linguistic analysis of suicide notes}

Regarding the analysis of the 23 suicide notes with LIWC, gender comparison showed that suicide notes written by women (n $=7 ; 30.44 \%)$ were longer $(p=0.018)$, with higher percentages of use of words with affective content $(p=0.033)$, positive feelings $(p=0.004)$ and positive emotions ( $p=$ $0.001)$. In feminine texts there were also higher percentages of verbs in past ( $p=$ $0.022)$ and future tenses $(p=0.027)$, spatial references $(p=0.010)$, negations $(p=0.033)$, tentative words $(p=0.012)$, pronouns in first person plural $(p=0.033)$ and punctuation marks $(p=0.022)$ (Table 2).

In the comparison by age, we only found a significantly higher use of punctuation particles in people younger than 65 years of age $(\mathrm{n}=14 ; 60.87 \%)$, with a mean proportion of use of exclamations of $2.40(\mathrm{SD}=2.18)$ vs. $0.48(\mathrm{SD}=1.02)$ in older $(p=0.009)$, and a mean proportion of use of commas of 4.13 $(\mathrm{SD}=3.92)$ vs. $0.68(\mathrm{SD}=1.54)(p=0.33)$ in older victims.

Comparing by habitat, rural inhabitants (n $=13 ; 56.52 \%$ ) used higher percentages of words referring to social processes ( $p=$ $0.049)$, whereas urban individuals used more negations $(p=0.030)$, numbers $(p=0.049)$, punctuation marks $(p=0.002)$ and words referring to positive emotions $(p=0.036)$, anxiety $(p=0.012)$, insight $(p=0.036)$ and time $(p=0.049)$ (Table 3). 
Table 1

Sociodemographic and forensic characteristics of note writers vs. non- writers.

\begin{tabular}{|c|c|c|c|c|c|}
\hline & \multicolumn{2}{|c|}{ Note writers } & \multicolumn{2}{|c|}{ Non writers } & \multirow[b]{2}{*}{$\mathrm{p}$} \\
\hline & $\mathrm{N}$ & $\%$ & $\mathrm{~N}$ & $\%$ & \\
\hline Sample & 26 & 18.06 & 118 & 81.94 & \\
\hline Gender & & & & & 0.707 \\
\hline Men & 18 & 69.23 & 86 & 72.88 & \\
\hline Women & 8 & 30.77 & 32 & 27.12 & \\
\hline Residence & Valid: 26 & & Valid: 105 & & 0.188 \\
\hline Rural & 14 & 53.85 & 71 & 67.62 & \\
\hline Urban $(>10.000)$ & 12 & 46.15 & 34 & 32.38 & \\
\hline Marital status & Valid: 25 & & Valid: 99 & & 0.065 \\
\hline Married/Couple & 7 & 28 & 48 & 48.48 & \\
\hline Single or similar & 18 & 72 & 51 & 51.52 & \\
\hline Method & Valid: 26 & & Valid: 116 & & \\
\hline Hanging & 15 & 57.69 & 66 & 56.90 & 0.885 \\
\hline Poisoning & 3 & 11.54 & 13 & 11.21 & 0.768 \\
\hline Fall & 2 & 7.69 & 13 & 11.21 & 0.862 \\
\hline Firearm & 3 & 11.54 & 12 & 10.35 & 0.862 \\
\hline Drowning & 2 & 7.69 & 7 & 6.04 & 0.895 \\
\hline Cut/pierce & 1 & 3.85 & 1 & 0.86 & 0.805 \\
\hline Struck & 0 & 0 & 2 & 1.72 & 0.805 \\
\hline Suffocation & 0 & 0 & 1 & 0.86 & 0.411 \\
\hline Electrocution & 0 & 0 & 1 & 0.86 & 0.411 \\
\hline Place of suicide & Valid: 23 & & Valid: 106 & & \\
\hline Home & 9 & 39.13 & 45 & 42.45 & 0.952 \\
\hline Annex & 11 & 47.83 & 28 & 26.42 & 0.076 \\
\hline Work & 1 & 4.35 & 26 & 24.53 & 0.061 \\
\hline Place with emotional content & 1 & 4.35 & 7 & 6.60 & 0.944 \\
\hline Institution & 1 & 4.35 & 0 & 0 & 0.399 \\
\hline Reported previous attempt & 5 & 19.23 & 23 & 21.70 & 0.808 \\
\hline Trigger & Valid: 19 & & Valid: 74 & & \\
\hline Mental illness & 3 & 15.79 & 40 & 54.05 & 0.006 \\
\hline Impairments in physical health & 1 & 5.26 & 14 & 19.92 & 0.274 \\
\hline Family troubles & 2 & 10.53 & 12 & 16.22 & 0.796 \\
\hline Emotional problems, separation or divorce & 9 & 47.37 & 2 & 2.70 & $<0.001$ \\
\hline Severe somatic disease & 2 & 10.53 & 6 & 8.11 & 0.902 \\
\hline Economic and/ or work trouble & 2 & 10.53 & 0 & 0 & 0.053 \\
\hline
\end{tabular}


Table 2

LIWC categories with significant differences in the percentage of words used by gender.

\begin{tabular}{|c|c|c|c|c|}
\hline LIWC categories & Statistics & Men & Women & $\mathrm{p}$ \\
\hline \multirow[t]{2}{*}{ Word count } & Mean (SD) & $86.38(111.33)$ & $288.85(169.95)$ & 0.018 \\
\hline & Median (min-max) & $32.50(7-390)$ & $323(14-509)$ & \\
\hline \multirow[t]{2}{*}{ 1st person plural (we, us, our) } & Mean (SD) & $0(0)$ & $0.25(0.28)$ & 0.033 \\
\hline & Median (min-max) & $0(0-0)$ & $0.27(0-0.77)$ & \\
\hline \multirow[t]{2}{*}{ Negations (no, not, never) } & Mean (SD) & $3(5.02)$ & $4.90(1.38)$ & 0.033 \\
\hline & Median (min-max) & $0.26(0-14.29)$ & $5.29(3.05-7.14)$ & \\
\hline \multirow[t]{2}{*}{ Affective processes (happy, cried) } & Mean (SD) & $5.14(4.54)$ & $8.02(1.88)$ & 0.033 \\
\hline & Median (min-max) & $4.70(0-15.38)$ & $7.14(6.19-11.45)$ & \\
\hline \multirow[t]{2}{*}{ Positive emotion (love, nice, sweet) } & Mean (SD) & $1.89(2.55)$ & $5.84(0.98)$ & 0.001 \\
\hline & Median (min-max) & $0.72(0-9.23)$ & $5.71(4.32-7.14)$ & \\
\hline \multirow[t]{2}{*}{ Positive feelings (happiness, love) } & Mean (SD) & $0.55(1.02)$ & $3.76(2.11)$ & 0.004 \\
\hline & Median (min-max) & $0(0-3.03)$ & $4.33(0-6.11)$ & \\
\hline \multirow[t]{2}{*}{ Tentative (maybe, perhaps, guess) } & Mean (SD) & $0.99(1.43)$ & $3.19(2.17)$ & 0.012 \\
\hline & Median (min-max) & $0.39(0-5.26)$ & $2.72(0-7.14)$ & \\
\hline \multirow[t]{2}{*}{ Past tense (went, ran, had) } & Mean (SD) & $0.94(1.58)$ & $2.84(1.98)$ & 0.022 \\
\hline & Median (min-max) & $0(0-5.41)$ & $3.37(0-4.91)$ & \\
\hline \multirow[t]{2}{*}{ Future tense (will, gonna) } & Mean (SD) & $0.48(1.29)$ & $1.51(2.52)$ & 0.027 \\
\hline & Median (min-max) & $0(0-5)$ & $0.54(0-7.14)$ & \\
\hline \multirow[t]{2}{*}{ Space (down, in, thin) } & Mean (SD) & $0.34(0.87)$ & $1.33(1.20)$ & 0.010 \\
\hline & Median (min-max) & $0(0-2.70)$ & $0.79(0-3.45)$ & \\
\hline \multirow[t]{2}{*}{ Punctuation marks (periods, commas) } & Mean (SD) & $4.61(4.77)$ & $13.64(10.38)$ & 0.022 \\
\hline & Median (min-max) & $4.50(0-13.59)$ & $10.87(1.53-28.57$ & \\
\hline
\end{tabular}

\section{Discussion}

Our sample of suicide victims was mainly composed of men, living predominantly alone in rural areas and with advanced age. The absence of gender differences in the method of suicide was noted, except in the case of suicide by gunshot (firearms only being accessible for hunting, a men hobby). The most commonly reported trigger was the existence of a prior mental illness. A worsening of the physical state was referred to as the main trigger in $16.13 \%$ of people who committed suicide. A similar trend was found in a psychological autopsy study conducted in the same population ${ }^{23}$ where a minor impairment of health status was considered the trigger in $26 \%$ of the cases analyzed (58\% older than 65 years old); whereas sentimental reasons were considered the main trigger of suicides in women between 35 and 55 
Table 3

LIWC categories with significant differences in the percentage of words used by habitat.

\begin{tabular}{lllll} 
LIWC categories & Statistics & Rural & Urban & $p$ \\
\hline Negations (no, not, never) & Mean (SD) & $2.02(3.09)$ & $5.62(4.93)$ & 0.030 \\
& Median (min-max) & $0(0-9.80)$ & $4.82(0-14.29)$ & \\
Numbers (second, thousand) & Mean (SD) & $0.22(0.60)$ & $1.43(2.45)$ & 0.049 \\
& Median (min-max) & $0(0-2.17)$ & $0.37(0-7.22)$ & \\
Positive emotion (love, nice, sweet) & Mean (SD) & $1.97(2.31)$ & $4.57(2.93)$ & 0.036 \\
& Median (min-max) & $1.43(0-6.87)$ & $4.96(0-9.23)$ & \\
Anxiety (worried, fearful, nervous) & Mean (SD) & $0.06(0.21)$ & $0.75(1.14)$ & 0.012 \\
& Median (min-max) & $0(0-0.76)$ & $0.26(0-3.57)$ & \\
Insight (think, know, consider) & Mean (SD) & $0.44(0.72)$ & $3.16(4.34)$ & 0.036 \\
& Median (min-max) & $0(0-2.42)$ & $1.94(0-14.29)$ & \\
Social processes (mate, talk, they) & Mean (SD) & $11.67(4.73)$ & $7.16(5.35)$ & 0.049 \\
& Median (min-max) & $11.76(5.26-20)$ & $6.83(0-14.67)$ & \\
Time (end, until, season) & Mean (SD) & $1.46(2.27)$ & $4.37(4.19)$ & 0.049 \\
Punctuation marks (periods, commas) & Mean (SD) & $3.33(3.81)$ & $12.60(8.98)$ & 0.002 \\
& Median (min-max) & $1.53(0-10)$ & $10.79(0-28.57)$ & \\
\hline
\end{tabular}

years. Consequently it has been suggested that focusing attention both at primary care and mental health services in people with advanced age who present a worsening in their general condition and in middle aged people reporting emotional difficulties would be rewarding for suicide prevention ${ }^{23}$.

The $18.06 \%$ of people in our sample left a suicide note, a percentage similar to previous reports in Spain $^{24}$ (between $10.50 \%$ and $18.70 \%)$.

In comparing suicide note writers vs. nonwriters there were significant differences in age, writers being younger than non-writers, a fact that could be related to emotional triggers and with educational level. The difference was magnified if we consider the gender of writers, with a mean age in women of
41.38 vs. 60.88 years in men, a fact that seems to enforce the influence of love affairs. Sentimental problems were far more frequent reported as trigger in suicide note writers, while mental illness was more frequent in non-writers. However, this variable should be observed with caution because it was subject to informant bias and it could not be collected in up to $35.42 \%$ of total cases. No significant differences were found in terms of the place of residence, although note writing seems more common in people living alone (separated, single, widowed), a result consistent with others previously reported ${ }^{3,5}$. Therefore within this sample, people who left suicide notes had a lower mean age, were less likely to be married or living with a couple, and the most frequent trigger was the existence of emotional problems. 
In the linguistic analysis of the notes, we found statistically significant differences by gender. The female suicide notes were considerably longer and contained a higher percentage of punctuation marks, which could indicate a more careful drafting and more interest in the transmission of information to others. Women used a higher percentage of words referring to positive feelings and emotions, more tentative and denials expressions (which introduce complexity in the text), more pronouns in the first person plural, and more verbs in past and future tenses. Some of these results (higher percentage of positive feelings and emotions and increased use of the first person plural) agree with those reported by Lester, Haines and Williams ${ }^{22}$, interpreted by the authors as evidence of greater involvement with others, moreover, the increased use of emotion words suggests a greater immersion in what is written. A study on the differences in the language used by gender ${ }^{25}$ concluded that female language is characterized by a higher percentage of verbs and words that relate to social and psychological processes, while male language would focus more on concerns relating to the present. Their results ${ }^{25}$ agree with our analysis where women use more verbs in past and future tenses, more first person plural (which has been associated with social integration) and use more emotion-related words and feelings, thus showing a more socio-emotional and dynamic writing style.

Regarding the absence of linguistic differences by age (except those relating to the use of punctuation marks), it has to be noted that our sample was very small and of a mature age $($ mean $=55.43$; $\mathrm{SD}=20.31)$, older than the samples described in other studies, (for instance in Lester et al. ${ }^{22}$; mean $=41.8$ ). This means that there was a shortage of young subjects to compare within our sample. An analysis of a sample of more than
3,000 participants ${ }^{26}$ comprising people from 8 to 70 found, as the life cycle progresses, a gradual increase in the percentage of use of positive emotion words, verbs in present and future tenses and words related to cognition, while showing a decrease in the percentage of negative emotion words, past tense verbs and references to themselves and others. Larger samples may be needed to find such differences in our community.

Finally, regarding place of residence we have found an increased use of punctuation marks in urban people (possibly related to a higher educational level) and a higher percentage of words indicating negation and insight (cognitive complexity indicators), positive emotions and anxiety (indicators of increased immersion in what is written), numbers and time references. Moreover, in rural areas there were a higher percentage of words referring to social processes. This category includes words relating to communication with others (talk, share...), friends and family (brother, friend...), and humans; it has been interpreted as a mark of social connection and integration $^{27}$, and could indicate further allusion to the reference group and loved ones in these notes (related to increased integration into community life that frequently occurs in rural areas), while social integration reflected in the writings would be lower in the urban group.

\section{Conclusions}

As we have seen, there were differences in our sample between those who left suicide note and those who did not. Note writers were younger, single, divorced or widowed, and sentimental troubles were reported as the most frequent trigger; whereas non- writers more often had a diagnosis of mental illness. 
The linguistic analysis of the suicide notes with LIWC showed differences in suicidal speech by gender, and added the innovative finding of linguistic differences according to the rural/ urban background of its authors. Suicide notes written by women showed more complexity and more interest in the transmission of information to others, while rural victims showed higher social integration in their suicide notes.

The present study is the first LIWC suicide notes analysis in Spanish and, as far as we know, the first one applied to suicide notes in Europe. We expect to continue this line of research extending the number of analyzed suicide notes and performing cross- cultural comparisons that allows us to determine the general features of the written suicidal speech, features that ideally would be useful for screening texts in an automated mode, with the aim of identifying at risk population and contribute to the prevention of suicide.

As limitations of our study, we must note the small sample size, the missing data in some of the cases studied, and the characteristics of the tool used, given that LIWC, being a quantitative tool, helps to avoid certain biases due to the researcher's theoretical approach, but it is unable to take into account contextual influences or figurative language.

\section{References}

1. Leenaars AA. Suicide across the adult life-span: An archival study. Crisis. 1989; 10(2): 132-51.

2. Shneidman ES. Suicide as psychache. Northvale NJ: Jason Aronson. 1993.

3. Callanan VJ, Davis MS. A comparison of suicide note writers with suicides who did not leave notes. Suicide Life Threat Behav. 2009; 39(5): 558-68.

4. Bhatia MS, Verma SK, Murty OP. Suicide notes: Psychological and clinical profile. Int J Psychiatry Med. 2006; 36(2): 163-70.
5. Haines J, Williams CL, Lester D. The characteristics of those who do and do not leave suicide notes: Is the method of residuals valid? Omega. 2011; 63(1): 79-94.

6. Foster T. Suicide note themes and suicide prevention. Int J Psychiatry Med. 2003; 33(4): 323-31

7. Leenaars AA. Suicide: A multidimensional malaise. Suicide Life Threat Behav. 1996; 26(3): 221-36.

8. Leenaars AA. Suicide notes in the courtroom. J Clin Forensic Med. 1999; 6: 39-48.

9. Lester D. Menninger's motives for suicide in genuine and simulated suicide notes. Percept Mot Skills. 1989; 69: 850 .

10. Menninger K. Man against himself. New York: Harcourt, Brace \& World. 1938.

11. Lester D, Seiden RH, Tauber RK. Menninger's motives for suicide in genuine, simulated and hoax suicide notes. Percept Mot Skills. 1990; 71: 248.

12. Pennebaker JW, Francis ME, Booth RJ. Linguistic Inquiry and Word Count (LIWC): LIWC2001. Mahwah, NJ: Erlbaum Associates. 2001

13. Ramírez-Esparza N, Pennebaker JW, García FA, Suriá R. La psicología del uso de las palabras: Un programa de computadora que analiza textos en Español (The psychology of word use: A computer program that analyzes texts in Spanish). Rev Mex Psicol. 2007; 24: 85-99.

14. Stirman SW, Pennebaker JW. Word use in the poetry of suicidal and nonsuicidal poets. Psychosom Med. 2001; 63(4): 517-22.

15. Lester D. Learning about suicide from the diary of Cesare Pavese. Crisis. 2009; 30(4): 222-24.

16. Lester D, McSwain S. Poems by a suicide: Sara Teasdale. Psychol Rep. 2010; 106(3): 811-12.

17. Lester D, McSwain S. A text analysis of the poems of Sylvia Plath. Psychol Rep. 2011; 109(1): 73-76.

18. Baddeley JL, Daniel GR, Pennebaker JW. How Henry Hellyer`s use of language foretold his suicide. Crisis. 2011; 32(5): 288-92.

19. Fernández-Cabana M, García-Caballero A, AlvesPérez MT, García-García MJ, Mateos R. Suicidal traits in Marilyn Monroe's Fragments. An LIWC Analysis. Crisis. 2013; 34(2): 124-30.

20. Handelman LD, Lester D. The Content of Suicide Notes from Attempters and Completers. Crisis. 2007; 28(2): $102-4$.

21. Lester D. Differences between genuine and simulated suicide notes. Psychol Rep. 2008; 103: 527-28. 
22. Lester D, Haines J, Williams CL. Content differences in suicide notes by sex, age, and method: A study of Australian suicide notes. Psychol Rep. 2010; 106 (2): 475-76.

23. García-Caballero A. Autopsia Psicológica. Cad Aten Prim. 2009; 16: 108-109.

24. Rodes F, Monera CE, Giner S, Martí JB. Notas suicidas. Rev Esp Med Leg. 1999; 23(86-87): 66-74.

25. Newman ML, Groom CJ, Handelman LD, Pennebaker JW. Gender differences in language use: An analysis of 14.000 text samples. Discourse Process. 2008; 45: 211-36.

26. Pennebaker JW, Stone LD. Words of wisdom: Language use over the life span. J Pers Soc Psychol. 2003; 85(2): 291-301.
27. Pennebaker JW, Lay TC. Language use and personality during crises: Analyses of Mayor Rudolph Giuliani's press conferences. J Res Pers. 2002; 36: 271-82.

\author{
* Corresponding author: \\ M. Fernández-Cabana \\ Department of Psychiatry \\ School of Medicine \\ University of Santiago de Compostela \\ Praza do Obradoiro \\ Santiago de Compostela 15782 \\ Spain \\ Phone number: 34-981-950901 \\ E-mail: mercedes.fcabana@gmail.com
}


